TRABAJOS DE PREHISTORIA

55, n. $^{\circ} 1,1998$, pp. 171 a 183

\title{
DÓLMENES EN LA CUENCA \\ DEL TAJO: RESTAURACIÓN Y CONSOLIDACIÓN DE MEGALITOS EN ALCÁNTARA (CÁCERES)
}

\author{
MEGALITHS IN THE BASIN OF THE TEJO \\ RIVER: RESTORATION AND \\ CONSOLIDATION OF MEGALITHIC \\ MONUMENTS IN ALCANTARA (CACERES)
}

\author{
PRIMITIVA BUENO RAMÍREZ (*) \\ RODRIGO DE BALBÍN BEHRMANN (*) \\ ROSA BARROSO BERMEJO (*) \\ M. ${ }^{a}$ AMPARO ALDECOA QUINTANA (*) \\ ANA BELÉN CASADO MATEOS (*)
}

\section{RESUMEN}

Se dan a conocer los primeros resultados de la excavación de dólmenes en el término de Alcántara (Cáceres), con el objeto de su consolidación y restauración. Se trata de arquitecturas en pizarra con el interés de poseer grabados megalíticos y materiales que indican la existencia de redes de intercambio en este sector del Tajo, tradicionalmente interpretado como marginal y prácticamente deshabitado en el transcurso del IV y III milenio a.C. Destaca la presencia de un ajuar campaniforme liso con vaso, cuenco con umbo y laminita metálica.

\begin{abstract}
We present the first results of the excavation of megaliths in the municipality of Alcántara (Cáceres), aimed to their consolidation and restoration. They are built of slate and possess materials and engravings that indicate the existence of exchange net works in this sector of the Tejo, which is traditionally interpreted as marginal and practically depopulated in the IV and III millennium b.C. Of note is the presence of a grave with plain Bell Beaker pottery, vessel and bowl dished omphalos base, and sheet metal.
\end{abstract}

(*) Área de Prehistoria. Universidad de Alcalá de Henares. C/ Colegios, 2. 28802 Alcalá de Henares. Madrid.

El artículo fue remitido en su versión final el 20-I-98.
Palabras clave: Cuenca del Tajo. Dólmenes de pizarra. Arte Megalítico. Campaniforme liso. Neolítico Final. Calcolítico. Redes de intercambio.

Key words: Basin of the Tejo. Megaliths in slate. Megalithic Art. Plain Bell Beaker. Late Neolithic. Chalcolithic. Exchange networks.

\section{INTRODUCCIÓN}

En el curso de 1997 la Junta de Extremadura y el Ayuntamiento de Alcántara promovieron una actuación en algunos de los dólmenes conocidos en ese término con el objetivo de ponerlos en valor desde el punto de vista turístico y arqueológico.

La acción afecta a seis monumentos y un menhir que forman parte de dos rutas monumentales propuestas por C. Montano y el Ayuntamiento de Alcántara. El volumen de la misma obliga a llevarla a cabo en un mínimo de dos campañas.

Los monumentos documentados en el término de Alcántara sobrepasan la treintena (Fig. 1) y son conocidos desde los años 70 gracias a la labor de prospección de Fernando Tostado. Fue él mismo quien años después dió a conocer la riqueza de Alcántara a Cleofé Rivero, quien excavó algunos monumentos, sin que conste referencia alguna so- 

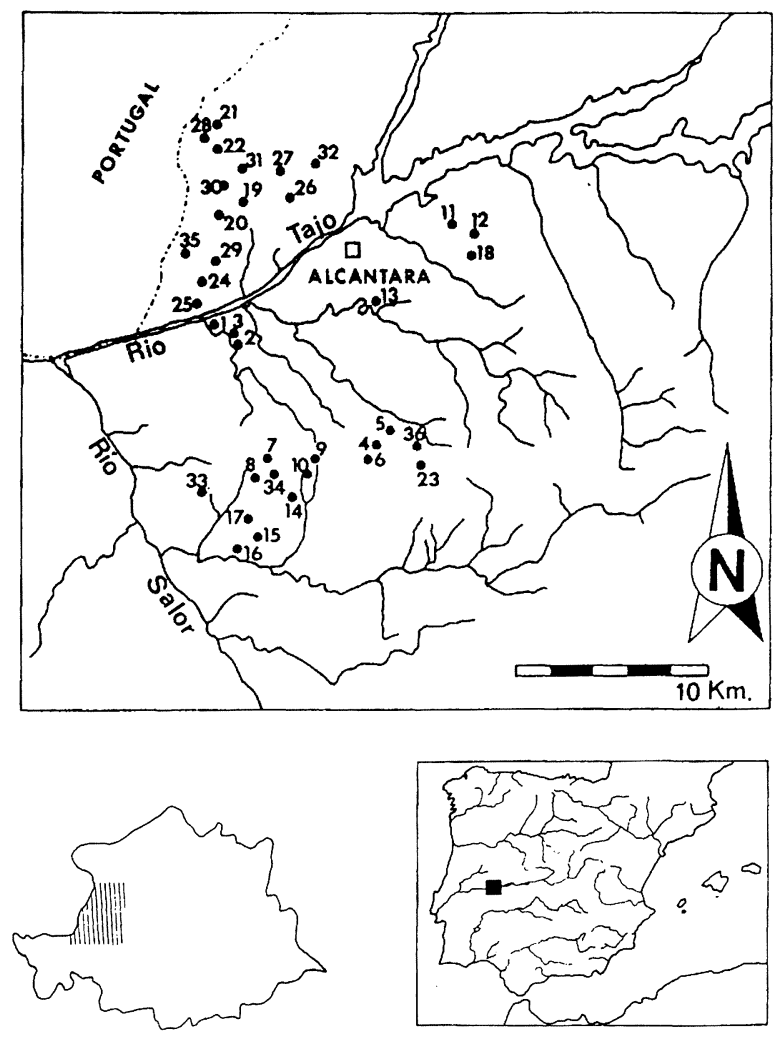

Fig. 1. Situación de Alcántara en la Península Ibérica y detalle de la ubicación de los monumentos a partir de plano de C. Montano (1987): 1. Trincones 1,2. Trincones 2, 3. Trincones 3, 4. Maimón 1, 5 . Maimón II, 6. Maimón 3, 7. Miras 1, 8. Miras 2, 9. Juan Rón 1, 10. Juan Rón 2, 11. Carrascal1, 12. Carrascal 2, 13. Fraila, 14. San Jordán, 15. Torre Oviedo 1, 16. Torre Oviedo 2, 17. La Fragua, 18. Belbís, 19. Retamar, 20. Carbonero, 21. El Pizarrón, 22. El Santo, 23. Camisones, 24. San Martín, 25. Calderones, 26. El Cabezo, 27. La Llorona, 28. Barcollero, 29. Cornejo, 30. Cerro Conejo 1, 31. Cerro Conejo 2, 32. Noguer, 33. Torre Botello, 34. Holmillo, 35. Moheda, 36. CasasViejas.

bre los resultados. Sólo disponemos de la mención de A. Guillén Oterino (1983) en un trabajo sobre muestras polínicas de la zona. Éstas se realizaron sobre los dólmenes del Retamar y el Pizarrón.

También Fernando Tostado nos los mostró en su día (Bueno Ramírez, 1987), al igual que a C. Montano (1987), quien realizó con ellos su Tesis de Licenciatura. Pese a conocerse el mencionado conjunto desde hace más de treinta años, los trabajos que aquí se presentan son los primeros realizados que ofrecen una documentación arqueológica contrastable.
Nuestros anteriores trabajos en Extremadura (Bueno Ramírez, 1984, 1987, 1988, 1989, 1994; Bueno Ramírez y Balbín Behrmann, 1991) nos permitían afrontar el análisis de estas arquitecturas con un conocimiento previo de su situación en la Extremadura española y en el contexto del Suroeste peninsular. Los trabajos de nuestro colega portugués J. de Oliveira (1994) en Cedillo (Cáceres) y en la zona portuguesa contigua, añadían valiosos datos para obtener una perspectiva mayor .

La primera campaña se desarrolló en julio de 1997 y, como la mayor parte de los trabajos arqueológicos, se debe al esfuerzo, colaboración y ánimo de muchas personas.

El Ayuntamiento de Alcántara proporcionó la infraestructura para un campo de trabajo de universitarios y, la Junta de Extremadura financió los informes arqueológicos previos a la restauración. El arqueólogo de la Junta, D. Javier Jiménez Ávila sẹ encargó de gestionar las condiciones de los trabajos de excavación y restauración y a él debemos en gran parte que se hayan podido realizar dignamente. Los dólmenes de Alcántara se restaurarán, pero además tendremos de ellos una buena documentación arqueológica.

El apoyo fáctico nos lo dieron los componentes del campo de trabajo, universitarios de Alcalá de Henares, Cáceres y Madrid que trabajaron muy duramente, por lo que queremos dejar constancia aquí de sus nombres: Raúl Albarrán Rodríguez, Miriam Alhambra Moreno-Arrones, Vanessa Becerra Sánchez, Isabel Borrego Pajuelo, $\mathrm{M}^{\mathrm{a}} \mathrm{Car}-$ men Borrego Pajuelo, Enrique Cerrillo Cuenca, $\mathrm{M}^{\mathrm{a}}$ Ángeles Cantillo Vázquez, Raquel Expósito Capilla, Jesús García Domínguez, Francisco Javier Heras Mora, Patricia Martín Cabello, Javier Galán Montano, Hitos Galán Montano, Verónica Domínguez Morales, Lourdes Gálvez Pérez, $\mathrm{M}^{\mathrm{a}}$ Soledad Galvez Pérez, Cristina García Moreno, Begoña Gómez Amado, Ana Belén González Salamanca, Ana Jardón Ruiz, Angel Luis Manso Iglesias, Jorge Mantilla González, Patricia Martín Barona, Víctor Martínez Palacios, Ángela $\mathrm{M}^{\mathrm{a}}$ Martínez Sempere, Armando Méndez Rubio, Cristina Navarro Martín, Yolanda Pereira Ramos, Francisco Javier Pello Sánchez, David Pérez Gil, Alicia Prada Gallardo, Roque Rojas Martín, Vanessa Bermejo Plata, Ainhoa Costa Fanega, Rocío Cisneros Carrasco, Sara Fernández Gracia, Nuria Requejo Lozoya .

Otras personas de la población colaboraron en la buena marcha del campo: José $\mathrm{M}^{\mathrm{a}}$ López Cal- 
vache, Clemente Montano, director del campo de trabajo, José $\mathrm{M}^{\mathrm{a}}$ Calvache $\mathrm{y}$, sobre todo, Concepción Tobías Cordobés, la cocinera que tan cariñosa fue con todos y de la que siempre recordaremos sus magníficas lentejas.

\section{CRITERIOS DE RESTAURACIÓN Y CONSOLIDACIÓN}

La restauración de monumentos megalíticos en la Península ha sido efectuada con diferentes criterios, según la Comunidad Autónoma en la que se han llevado a cabo los trabajos, según el presupuesto en ellos invertido y, desde luego, según la época en que ésta haya sido realizada (Cruz, 1988; Delibes et alii, 1993)

La asociación de arqueólogos y arquitectos sólo se da en el caso de que el proyecto disponga de una inversión importante, pues los arquitectos cobran por obra, mientras que si la inversión es mínima, es el arqueólogo el que se hace cargo de la restauración. Este es el caso de la consolidación de los dólmenes de Alcántara.

Todas las restauraciones recientes insisten en un respeto máximo hacia las estructuras antiguas, manteniendo los materiales originales y la situación de los mismos. Dicha situación se conoce a través del necesario informe arqueológico previo a cualquier movimiento de tierras que se efectúe en el monumento.

Por tanto, la primera premisa es disponer de información arqueológica sobre la situación de los componentes arquitectónicos para no inventar nada, sino reponer lo que ya existe, en todo caso, recolocando los ortostatos movidos por la presión de las tierras o por la acción humana reciente. Si resulta necesario recrecer alguna zona por cuestiones de estabilidad, ésta se marca de modo diferente.

Nuestro trabajo ha tenido en cuenta estas consideraciones y ha hecho especial hincapié en la descripción exhaustiva de cada una de las actuaciones de la restauración, con el objeto de que esta información pueda ser útil para trabajos similares o para posteriores investigaciones sobre el mismo monumento. Dicho informe quedará en la oficina correspondiente de la Junta de Extremadura y en el Ayuntamiento de Alcántara.

Como norma general, nos hemos limitado a poner en pie los ortostatos caídos, resituar los que disponían de una fosa contrastable por la informa- ción arqueológica y recrecer el túmulo para acercarnos al volumen real del monumento.

El objeto último es que el espectador consiga una imagen lo más próxima posible de lo que fue el monumento, del lugar que ocupaba en el espacio y de lo que suponía como intervención humana en el territorio. Toda idea equivocada que demos de ello, será la que reciba el espectador e integre como real, contribuyendo así a su desinformación.

Un capítulo fundamental en este tipo de proyectos de consolidación y restauración es la información gráfica que se adjunte. Esta debe ser básicamente de dos tipos: la fija que se hace constar en cartelería in situ y la móvil, es decir trípticos, guías breves y publicaciones especializadas. La información de cartelería ha de ser concisa y tremendamente visual, mientras que la información que puede llevar consigo el espectador interesado debería reflejar detalles arquitectónicos, análisis de los ajuares, propuestas cronológicas y culturales.

Ambos tipos de información se encuentran en este momento en trámites por parte de la Junta de Extremadura, aunque esperamos se concrete en breve la normativa para incorporarla en nuestra segunda campaña

\section{TRABAJOS ARQUEOLÓGICOS DE LA PRIMERA CAMPAÑA. JULIO 1997}

Los monumentos sobre los que se ha actuado en esta campaña se sitúan a los lados de la carretera Alcántara-Membrío y ya eran conocidos.

Para afrontar el trabajo establecimos dos equipos pues debíamos cumplir unos objetivos mínimos bastante amplios en muy poco tiempo, por cuestiones presupuestarias. Excavamos tres monumentos, dos de ellos mejor conservados: Maimón II y Juan Rón I y, otro en peores circunstancias arquitectónicas, pero con abundante material: Maimón I.

Este último conservaba cuatro ortostatos de pizarra, mermados en su altura original, y escasos restos de túmulo (Bueno Ramírez, 1987, 1994: fig. 24; Montano, 1987: fig. 2).

El planteamiento de la excavación se dirigía a localizar los ortostatos que completasen la delimitación de la cámara, la posible existencia de corredor y los restos de la estructura tumular.

Utilizamos como metodología de excavación la delimitación de cuadrículas y el levantamiento de niveles cada $5 \mathrm{~cm}$. con acotamiento de cada una 
de las piezas que de este modo se van situando en los sucesivos levantamientos planimétricos.

Sólo documentamos un ortostato más de la cámara que permitía identificar ésta como una estructura circular, quizá abierta hacia el Sureste y fosas que indicaban la probable existencia de más piezas, hoy desaparecidas. Todos los ortostatos de la cámara habían sido insertados en la pizarra base del terreno, mediante una profunda excavación previa que en algunos casos, casi llegaba al metro. No localizamos nada que pudiera interpretarse como corredor.

Lo que quedaba de túmulo se dibujaba como un estrecho anillo adosado a los ortostatos de la cámara, aunque es de suponer que el resto ha desaparecido con los trabajos agrícolas (Fig. 2).

El sistema constructivo queda claro en el alzado del monumento (Fig. 3). Se ha excavado una fosa en la pizarra del terreno aprovechando un sector más alomado sobre un pequeño montículo. En esta zanja inicial se han introducido los ortostatos y sus calzos, además de las primeras hiladas del anillo tumular. De este modo se consigue una construcción absolutamente compacta, en la que la primera hilada del túmulo es tambien el contrafuerte de la cámara. Este sistema pese a no ser muy común, se ha documentado en algunos megalitos alentejanos como el recientemente excavado de Belhoa (Gomes, 1997).

El material comenzó a aparecer prácticamente en superficie por lo que es asombroso que el monumento haya llegado hasta nosotros. La profundidad entre el suelo actual y la pizarra del terreno es de $30 \mathrm{~cm}$. Todo este relleno poseía materiales pertenecientes al sepulcro.

Entre todo el ajuar destacaríamos una placa de arenisca de $25 \mathrm{~cm}$. con huellas notorias de pintura roja (Lám. I). Otra placa, esta vez en esquisto, con cabeza destacada y decoración de fajas de triángulos, tipo B4 (Bueno Ramírez, 1992: 556) que apareció junto a la anterior (Fig. 4). El material lítico se compone sobre todo de puntas de flecha de factura muy cuidada en sílex y una llamativa lámina en sílex blanco de $19 \mathrm{~cm}$. de longitud.

Algunas de las piezas líticas se localizaron al interior de las fosas realizadas para levantar el monumento, luego pertenecen al momento de fundación del mismo.

En la misma finca se conocen restos de otros monumentos. Uno de ellos es el de Maimón II. Aparentemente se encontraba más completo que

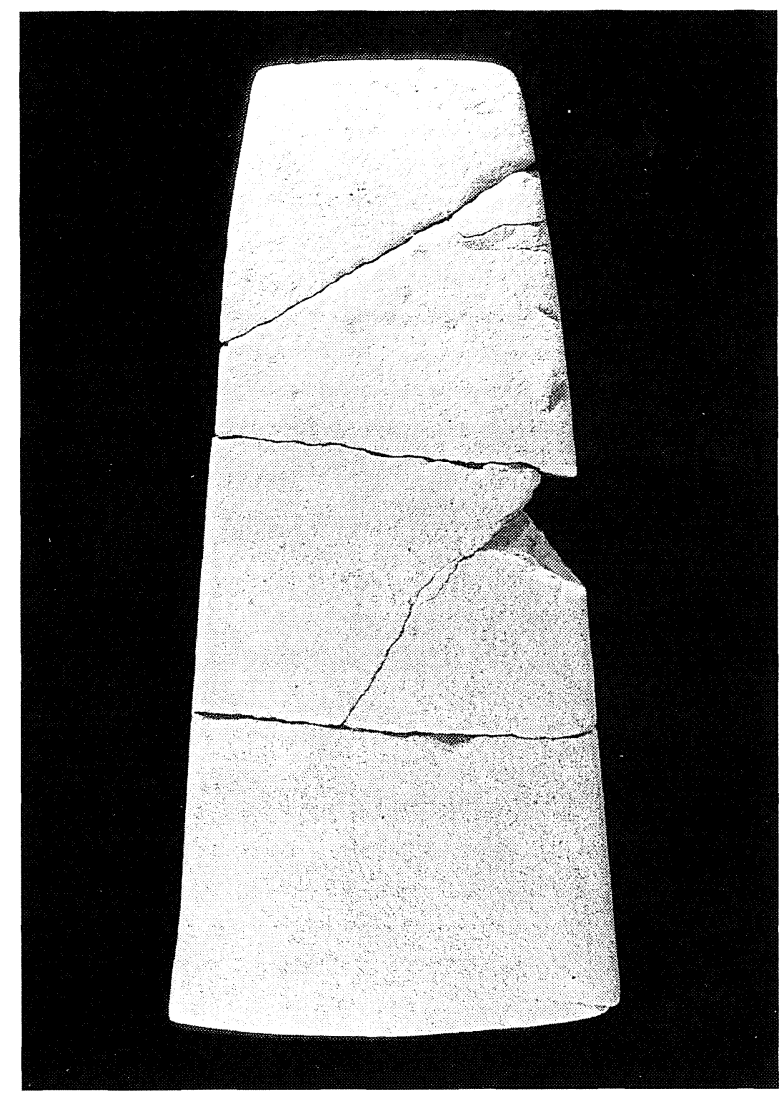

Lám. I. Placa de arenisca con someros piqueteados y restos de pintura roja del dolmen de Maimón I. (Foto R. de Balbín).

el anterior (Bueno Ramírez, 1987; 1994, fig. 25; Montano, 1987: fig. 2) y se dibujaba como una cámara con corredor y túmulo delimitado.

Es una arquitectura de pizarra con una cámara de $2,30 \mathrm{~m}$. de diámetro y una altura en torno al metro. La mayor parte de los ortostatos que componen la cámara están grabados (Láms. II y III).

La excavación se planteó para documentar la totalidad de la estructura, túmulo incluido. En ella pudimos localizar algunas piezas de la cámara, desplazadas de lugar o caídas al interior, lo que nos permitió incluirlas en la restauración y conocer el número total de componentes de la cámara: 14 (Figs. 5 y 6).

El corredor estaba bien conservado y la sola decoración localizada está en el único ortostato de cobertura recuperado durante la excavación.

El túmulo tiene $12 \mathrm{~m}$. de diámetro y está muy bien conservado. Es una compacta masa de pizarra y tierra, delimitada por piezas tambien esquis-

T. P., 55, n. $^{\circ} 1,1998$ 


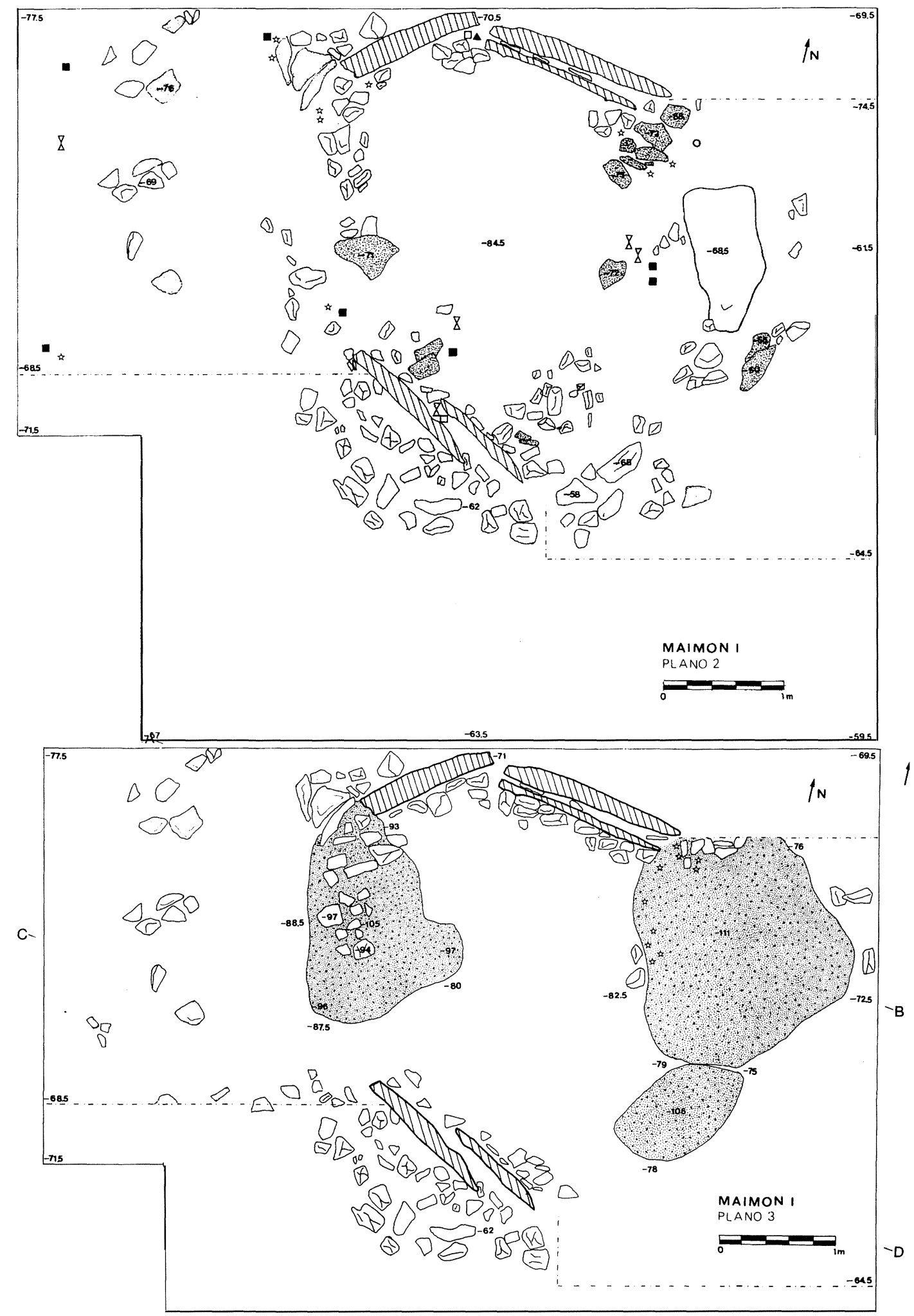

Fig. 2. Plantas del dolmen de Maimón I. Plano 2: situación de los hallazgos del nivel de base. Plano 3: delimitación de las fosas que alcanzan el anillo posterior del túmulo 


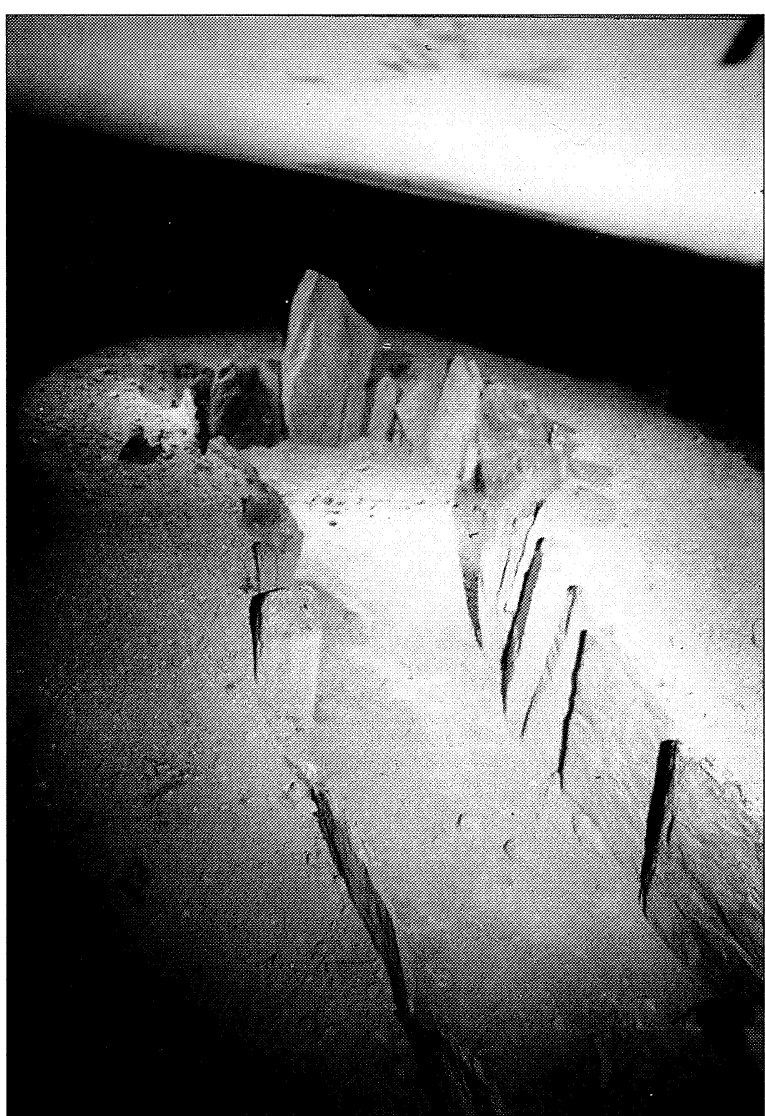

Lám. II. Vista del monumento de Maimón II ya restaurado. (Foto R. de Balbín).

tosas colocadas verticalmente. Sólo el cuadrante Noroeste fue afectado por una ocupación reciente, quizá una cabaña.

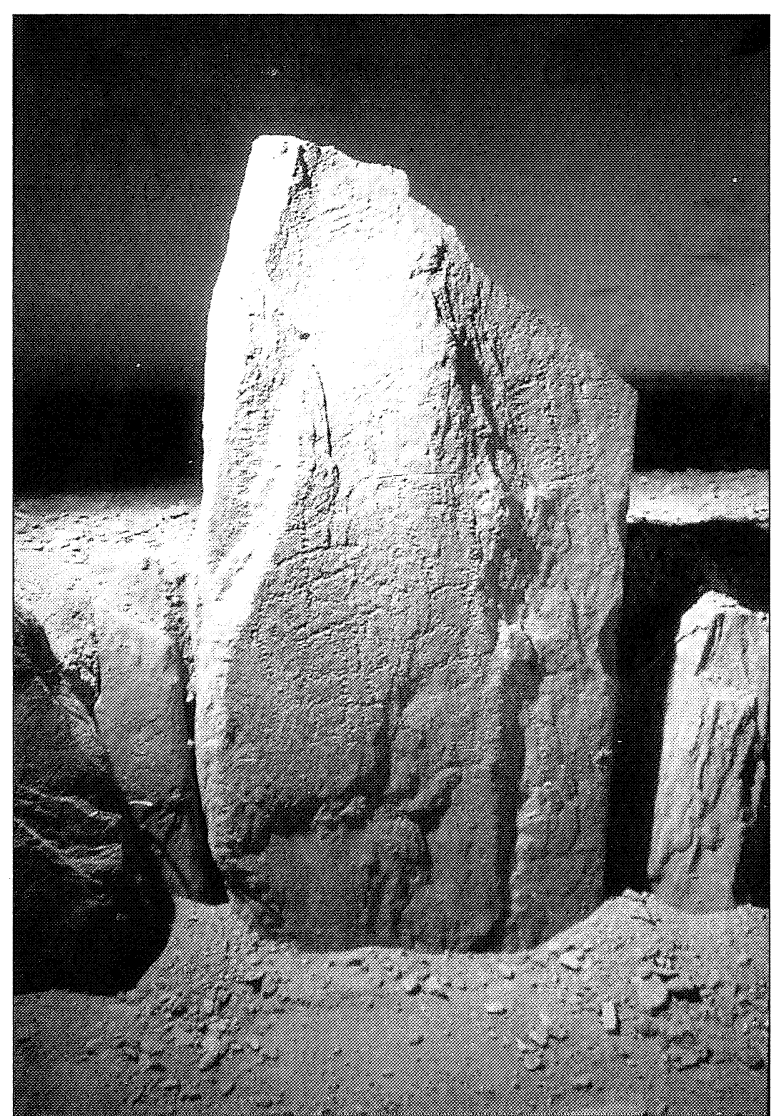

Lám. III. Detalle de los grabados del ortostato de cabecera del dolmen de Maimón II. (Foto R. de Balbín).

Como siempre, excavamos en niveles artificiales de $5 \mathrm{~cm}$. Sólo en la base registramos restos del nivel original en el que aparece un ortostato caído,
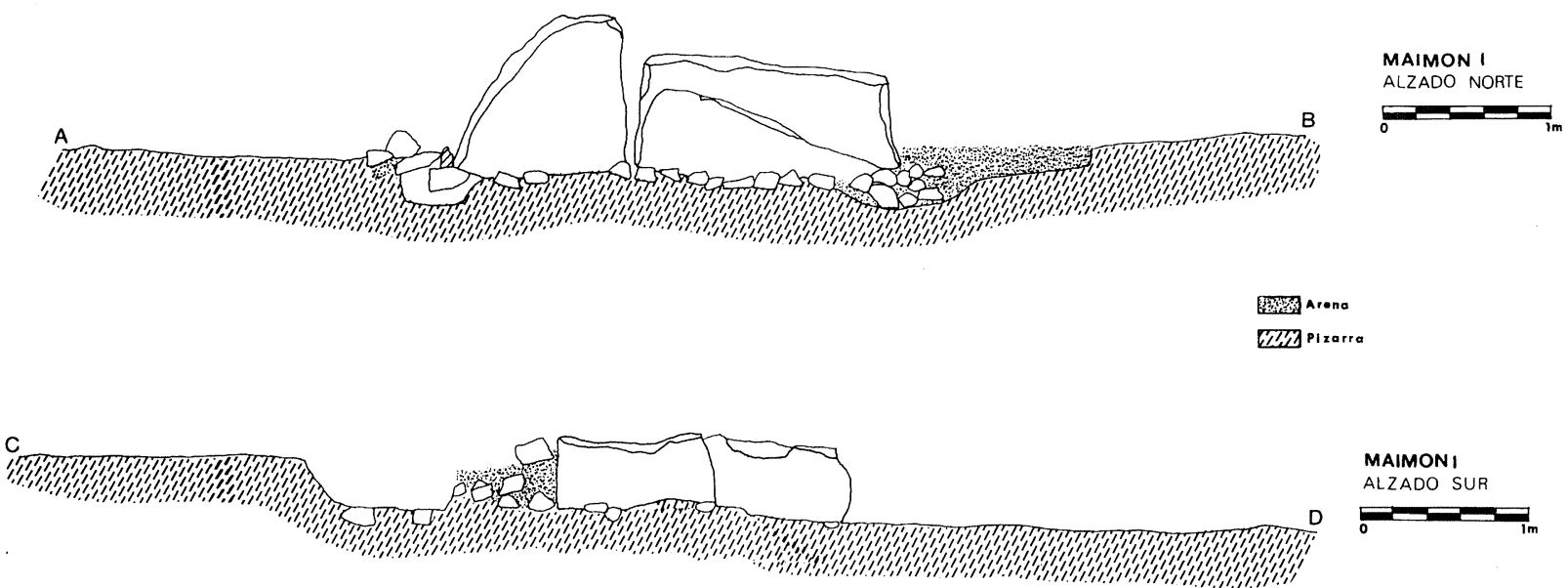

Fig. 3. Alzados Norte y Sur del dolmen de Maimón I.

T. P., 55, n. ${ }^{\circ} 1,1998$ 


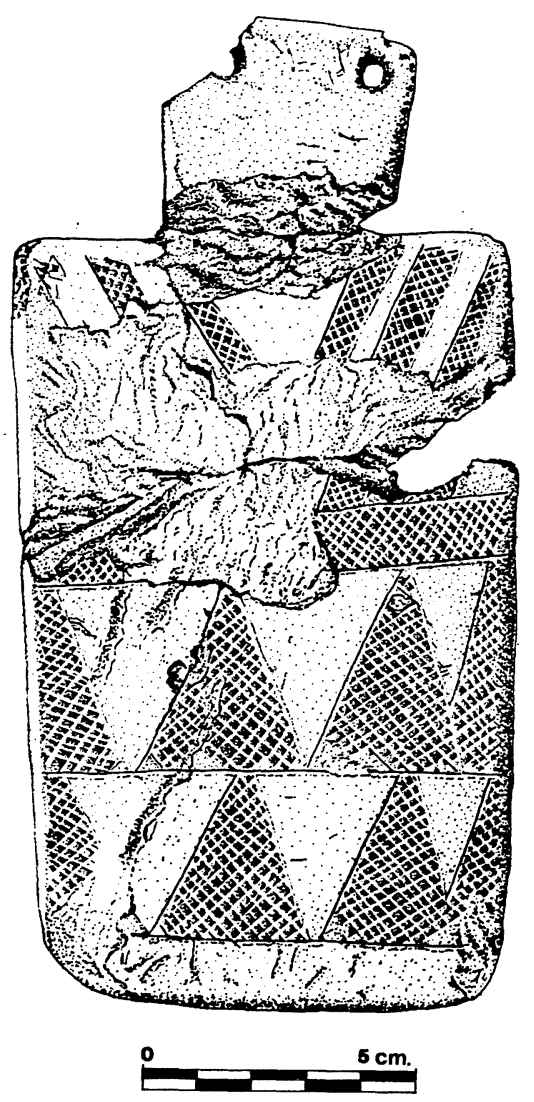

Fig. 4. Placa tipo B4 de Maimón I.

que, una vez levantado, se muestra absolutamente cubierto de grabados. También en este nivel documentamos un cuenco entero in situ y abundantes restos líticos en pizarra.

En una finca muy próxima, se encuentra el monumento de Juan Rón I. Todos los dólmenes documentados se encuentran muy relacionados espacialmente. Visualmente, desde Juan Rón I se controlan los dos de Maimón y desde Maimón II, el I y el de Juan Rón I. Maimón I queda entre los dos, en una cota algo más baja.

Juan Rón I aparecía como una cámara muy cerrada, con corredor (Bueno Ramírez, 1987, 1994: fig. 23; Montano, 1987: fig. 3).

No se apreciaba prácticamente el levantamiento del túmulo. Nuestra excavación reveló un corredor desarrollado del que aún se conservaba alguna pieza de cobertura que en la restauración se colocó en su lugar.

Todo el monumento se ha construido en pizarra, pero las dimensiones son sensiblemente mayores a las mencionadas para Maimón II. Tiene
2,30 m. de diámetro, $1,50 \mathrm{~m}$. de altura. El corredor $5 \mathrm{~m}$. de desarrollo y $0,90 \mathrm{~m}$. de altura máxima. El túmulo está practicamente arrasado aunque en el sector Este aún pudimos localizar parte de su estructura formada por piedras medianas compactadas con tierra. No pudimos confirmar si estaba delimitado como el de Maimón II.

La cámara había sido violada de antiguo, lo que explica la situación forzada de algunos de sus ortostatos. Aún así, próximo al nivel original pudimos documentar varias puntas de flecha, una gran placa con cabeza destacada y decoración de fajas de triángulos rellenos, tipo B4 (Bueno Ramírez, 1992: 576) y algunas cuentas de collar.

El corredor, a partir de $1,40 \mathrm{~m}$. del punto 0 estaba intacto. La ocupación más reciente es campaniforme. Los responsables de esta utilización dejaron su huella en el sector del corredor más próximo a la cámara con una piedra colocada transversalmente al decurso del mismo, con idea probablemente de delimitar una especie de "cista". En este lugar localizamos un cuenco con umbo, un vaso y una lámina metálica, es decir un ajuar campaniforme clásico, con la particularidad de que los vasos no estaban decorados (Figs. 7 y 8 ).

Bajo este nivel, todo el suelo del corredor estaba cubierto de vasos completos, hachas pulimentadas, cuentas de collar, colgantes (Fig. 9) y algunas puntas de flecha. Uno de los ortostatos del corredor muestra un grupo de cazoletas profundamentemente excavadas (Lám. IV). Parte del material que acabamos de describir apareció literalmente incrustado en las cazoletas, lo que ratifica la contemporaneidad de materiales y decoración (Bueno Ramírez y Balbín Behrmann, 1997b).

\section{DÓLMENES DE ALCÁNTARA, UNA NUEVA APORTACIÓN A LA CONSIDERACIÓN DE LOS MEGALITOS DE LA CUENCA DEL TAJO}

Una sola campaña en los dólmenes de Alcántara nos ha permitido documentar una riqueza en las formas arquitectónicas, y una variedad en las materias primas que nos llevan a vislumbrar una región en época Neolítico/Calcolítico de una entidad hasta ahora insospechada.

Materiales como la jadeíta o el azabache suponen que este sector, hoy casi desértico de las orillas del Tajo, tuvo en el IV y III milenio a.C. un protagonismo que hasta el momento desconocía- 


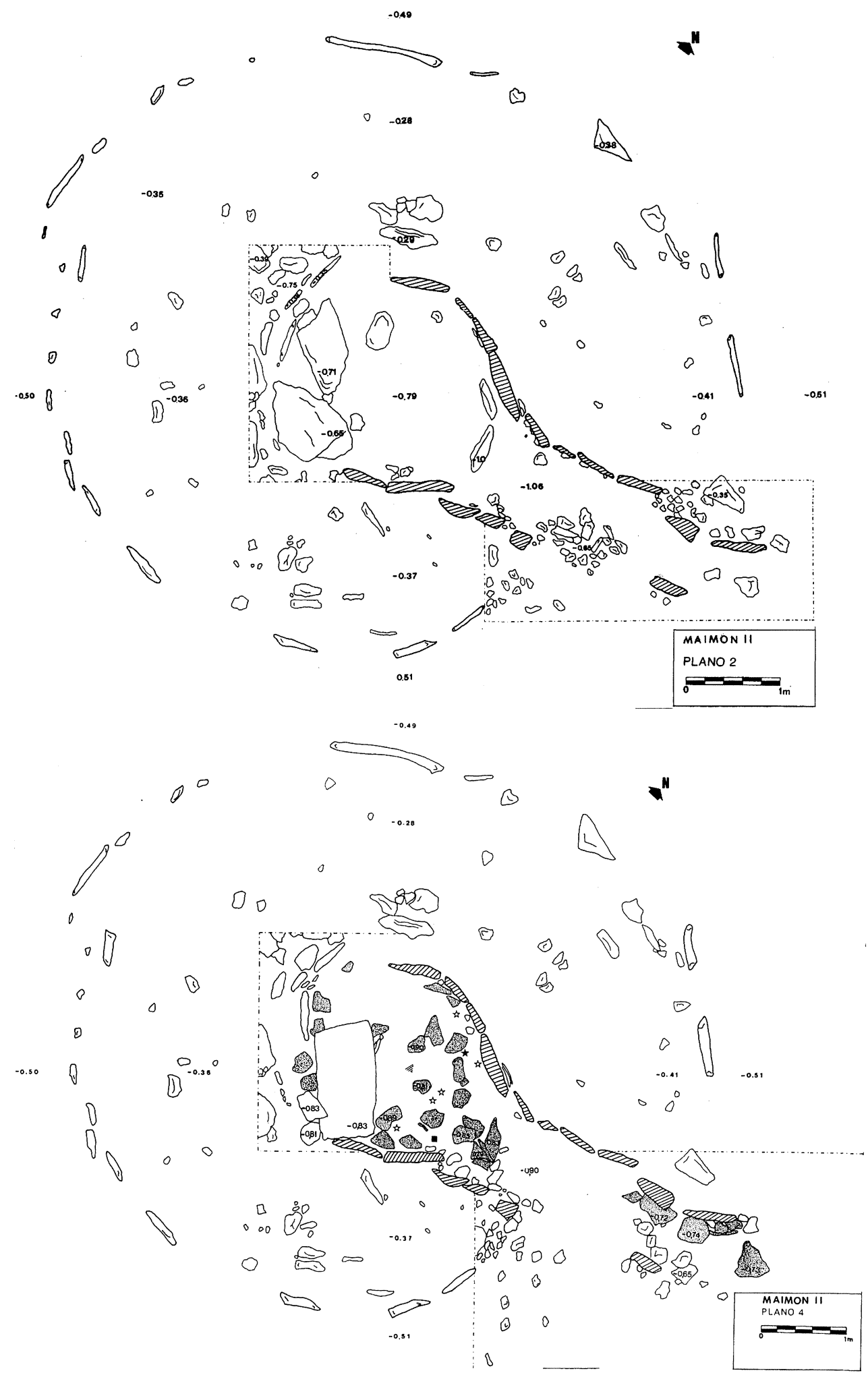

Fig. 5. Plantas de Maimón II. Plano 2: situación de pidras caídas en la cámara. Plano 5: ubicación de los hallazgos del nivel de base; en punteado, situación de las fosas de los ortostatos reimplantados.

T. P., 55, n. $^{\circ} 1,1998$ 


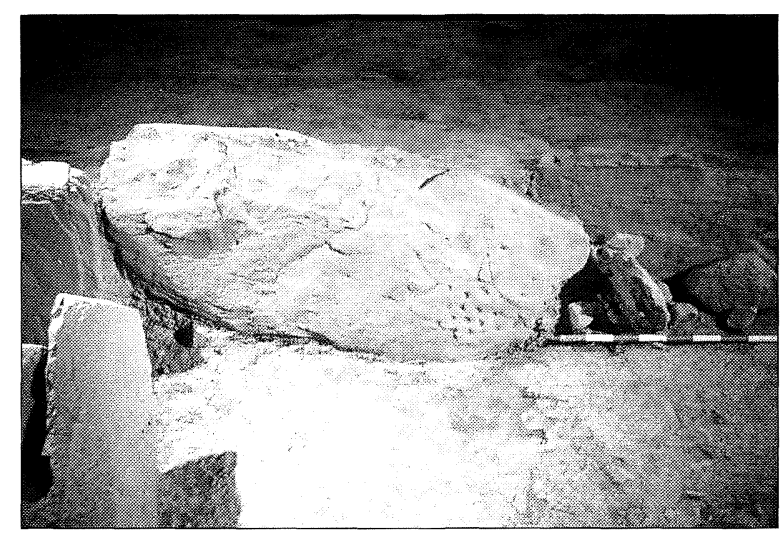

Lám. IV. Ortostato decorado del corredor del dolmen de Juan Rón I. (Foto R. de Balbín).

mos. Lo lógico sería que la continuación de nuestros trabajos, además de más sepulturas, nos llevara a la documentación de algún poblado importante, pues todo lo que hemos visto en los depósitos sepulcrales ha debido ser generado por algún tipo de excedente.

La presentación que hacemos ahora de los resultados es un pequeño avance y, por tanto, no podemos detenernos demasiado en comparaciones y paralelos que nos dibujarían la presencia de una amplia cultura alentejana (Bueno Ramírez, 1988: 195-198). Pero estas líneas son suficientes para destacar dos hechos que consideramos culturalmente de gran trascendencia: la presencia de Arte Megalítico y la contemporaneidad de formas ar- quitectónicamente diversas de un modo semejante a ambos lados de la frontera actual.

La decoración completa de Maimón II y la del corredor de Juan Rón I vienen a incidir en el convencimiento que hemos manifestado en diversas ocasiones de la extensión delArte Megalítico en la Península Ibérica (Bueno Ramírez y Balbín Behrmann, 1992, 1995, 1997 a y b; Balbín Behrmann y Bueno Ramírez, 1996). El estado de las piezas de la cámara de Juan Rón I y de Maimón I, cuya materia prima es un esquisto muy deleznable nos permite incluso sugerir, la posibilidad de que en origen también hubiesen tenido decoración.

Los grabados de Maimón II son piqueteados anchos, de poca profundidad. Los temas de casi todos los ortostatos, a excepción del frontal, son predominantemente circulares. Sólo el que se sitúa a la entrada de la cámara, en su lateral norte, parece de caracter animalístico. Como en otras decoraciones tótales, el frontal de la cámara destaca por la mayor cantidad de decoración y por la situación preferente del tema antropomorfo (Bueno Ramírez y Balbín Behrmann, 1994, 1996b). En este caso se trata de ramiformes esquemáticos.

El estilo de los grabados tiende a un cierto abigarramiento. Las formas se mezclan unas con otras recordando sobremanera el estilo más común en los elementos al aire libre. No olvidemos que nos encontramos en una zona muy próxima a los grabados del complejo del Tajo (Batista, 1981; Gomes, 1983), de los que tenemos representación
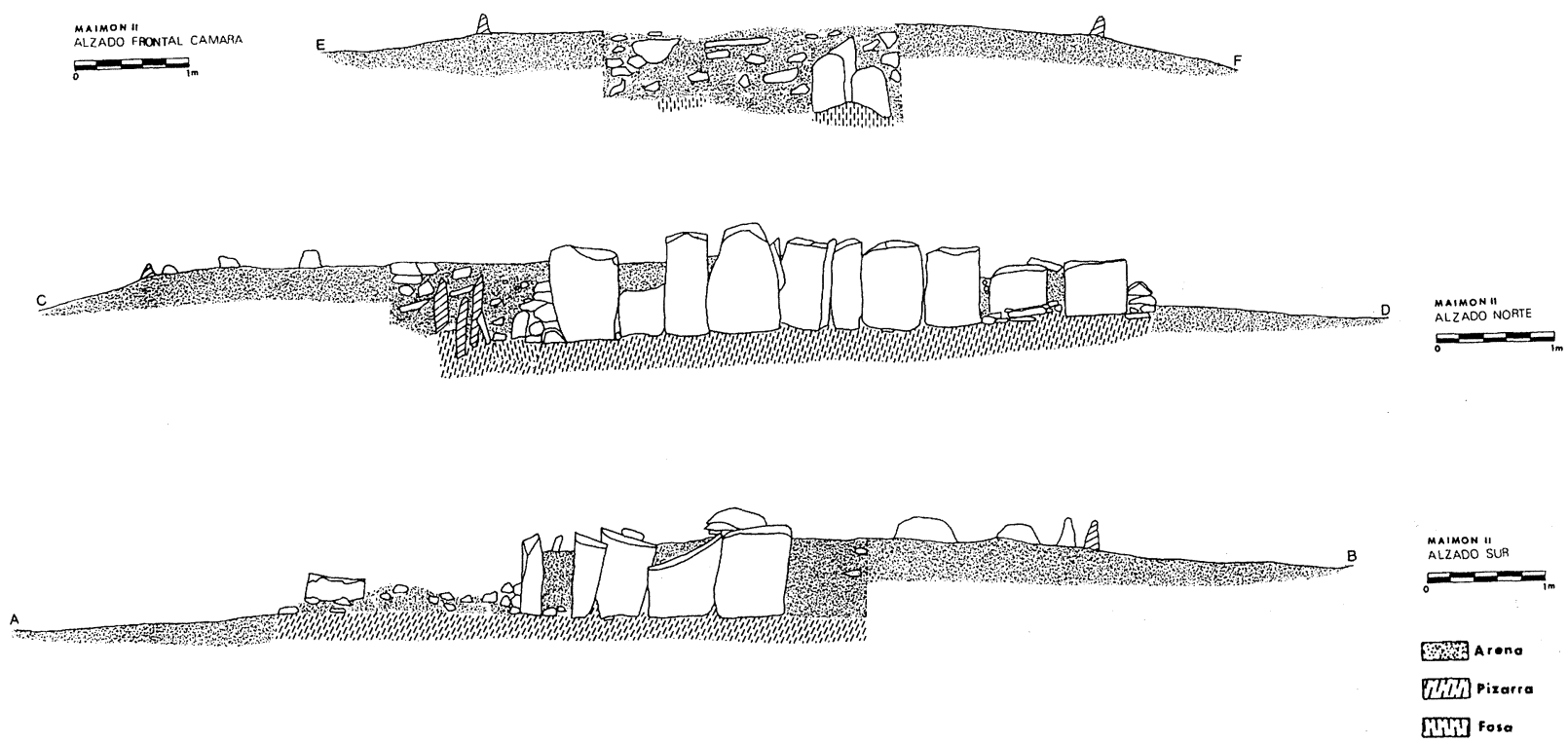

Fig. 6. Alzados del dolmen de Maimón II. 


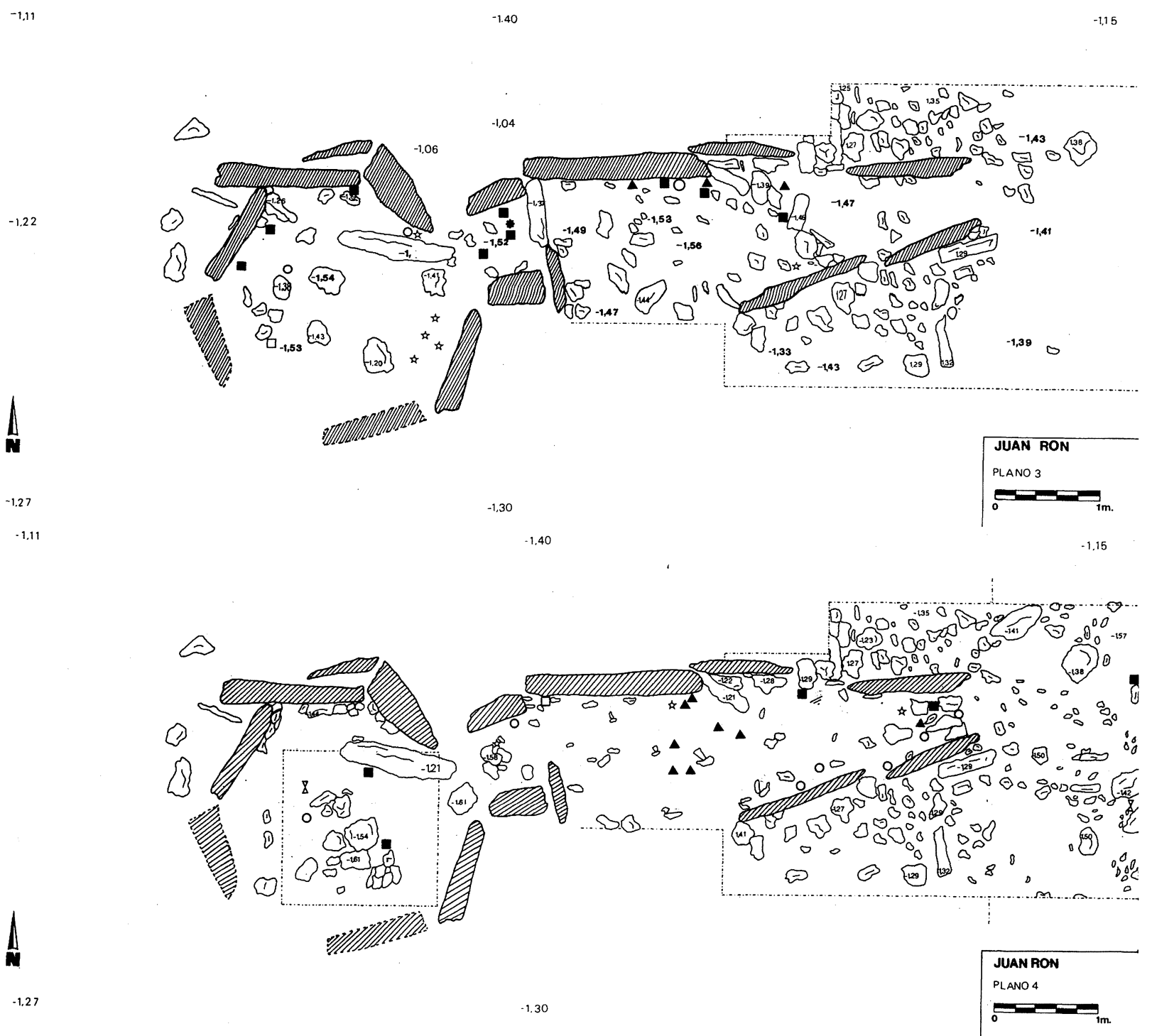

Fig. 7. Plantas del dolmen de Juan Rón I. Plano 3: situación de los hallazgos en el nivel con campaniforme liso. Plano 4: hallazgos en la base del monumento.

en el mismo término deAlcántara (Montano y Domínguez, 1988)

La existencia de grabados en el techo del corredor se da igualmente en otros monumentos sureños, como el dolmen de Soto I (Balbín Behrmann y Bueno Ramírez, 1996) y el recientemente descubierto de Alberite I, en Cádiz (Bueno Ramírez y Balbín Behrmann, 1996a)

Ya se conocían grabados y pinturas en dólmenes extremeños (Balbín Behrmann y Bueno Ramírez, 1981; Bueno Ramírez, 1988; Bueno Ramírez y Balbín Behrmann, 1992, 1997a). Creemos que los grabados de Alcántara son un elemento más que nos permite augurar una presencia de decoraciones mucho más masiva que la hasta ahora documentada. Quizá revisiones exhaustivas de dólmenes semejantes a uno y otro lado de la frontera darían nuevos elementos de análisis.

Por tanto, uno de las primeras conclusiones de carácter cultural que podemos extraer de nuestro trabajo en Alcántara es que esta zona tambien conoció el Arte Megalítico, confirmando la extensión de éste a toda la Península y, desde luego, al sector suroccidental (Bueno Ramírez y Balbín Behrmann, 1997a y b).

Todas las arquitecturas documentadas son de 

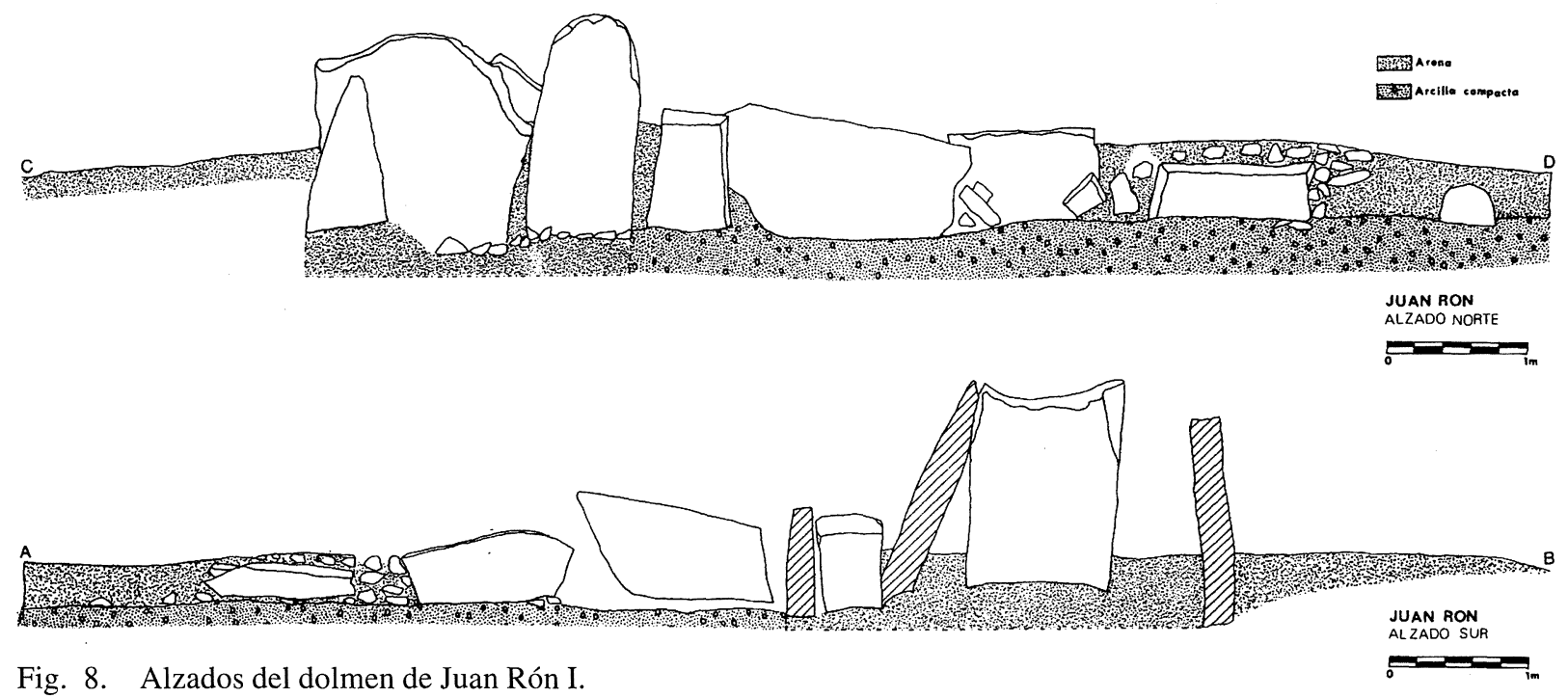

Fig. 8. Alzados del dolmen de Juan Rón I.

pizarra, material poco común aunque existente, en los conjuntos megalíticos occidentales. El interés del uso de esta materia prima estriba, para la zona extremeña, en que se han realizado del mismo modo arquitecturas de mayor y menor envergadura (Bueno Ramírez, 1988: 188, 1994).

Maimón II y Juan Rón I se manifiestan como cámaras con corredor. Maimón I aparenta ser una cámara de diámetro considerable, quizá sin corredor o con corredor corto. Maimón II es de poca altura y diámetro tumular discreto y Juan Rón I es de mayor altura y aunque no conocemos su diámetro tumular, podemos sospechar que debía ser mayor a los casi $8 \mathrm{~m}$. del desarrollo total del monumento.

Los materiales documentados nos conducen grosso modo a los momentos de transición entre Neolítico Final/Calcolítico, tan bien conocidos en este sector del Suroeste peninsular. Cuando finalicemos el estudio detallado de los mismos, es posible que podamos afinar más pero, en principio, todos los monumentos han sido utilizados en momentos similares. Este polimorfismo en las necrópolis megalíticas ibéricas posiblemente exista en fechas antiguas (Bueno Ramírez, 1994; Jorge, 1990), aunque algunos autores sólo lo consideran factible en fechas más recientes (Cruz, 1988).

El estudio de los materiales propone cuestiones de gran interés y hasta cierto punto inéditas. Este es el caso de la abundancia de piezas en pizarra: puntas de flecha, alabardas, hojas.

Conocíamos la existencia de industria de pizarra de modo marginal en megalitos extremeños
(Bueno Ramírez, 1988) y, desde luego, en poblados al aire libre de la zona del Guadiana (Enríquez, 1990), pero no en la cantidad y variedad que hemos detectado en nuestras excavaciones en Alcántara.

Los monumentos alcantarinos excavados en esta campaña pueden situarse en momentos recientes del desarrollo del megalitismo peninsular, pero habremos de esperar a otras campañas para proponer una secuencia más detallada, pues creemos que muchas de las sepulturas del sector pueden catalogarse entre los tipos más pequeños que ya hemos documentado en otras zonas extremeñas (Bueno Ramírez, 1994: 30-55). Esto supondría no sólo la contemporaneidad de arquitecturas de tamaño medio o grande, como las ahora presentadas, sino la contemporaneidad de éstas con formas aún más pequeñas que quizá indiquen algún tipo de diferenciación social. Desde luego, los ajuares documentados hasta el momento son ricos en objetos de prestigio y, muy especialmente, los de Juan Rón I y Maimón I.

Es demasiado pronto para pronunciarnos, pero nos parece interesantísima la oportunidad de plantearnos este tipo de preguntas en un sector tan poco documentado del megalitismo peninsular que, incluso, se había llegado a considerar absolutamente marginal a los conjuntos de gran envergadura documentados al otro lado de la frontera.

Recientes investigaciones en Portugal llevan a las mismas cuestiones: la existencia de arquitecturas en pizarra, implantadas en territorios pobres desde el punto de vista agrícola y que presentan 


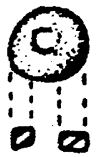
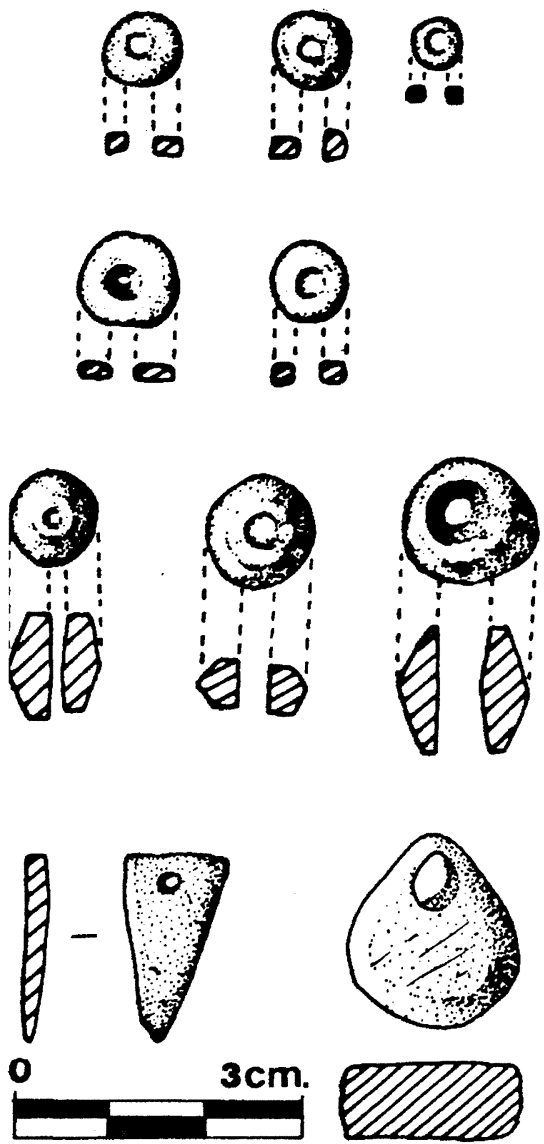

Fig. 9. Cuentas y colgantes del dolmen de Juan Rón I.

envergaduras diferentes (Calado, 1994; Oliveira, 1990). Ya G. Leisner (1949) había reparado en estos monumentos de pizarra y señalado sus diferencias volumétricas, de ajuar y de territorio.

Afortunadamente, hoy día, los contactos entre arqueólogos portugueses y españoles, tan necesarios para emprender proyectos conjuntos en zonas de frontera, son abundantes. En la actualidad estamos preparando un proyecto de estudio de las culturas megalítícas en el Tajo Internacional en el que intervienen los Ayuntamientos de uno y otro lado de la frontera, los arqueólogos portugueses y españoles que trabajamos a ambos lados y las instituciones implicadas. Esperamos que proyectos como éste, nos sirvan para conocer mejor nuestras culturas más antiguas, además de para acrecentar las relaciones con nuestros colegas portugueses porque nos necesitamos mutuamente para comprender el entramado cultural de los conjuntos prehistóricos situados a uno y otro lado de una frontera tan reciente.

\section{BIBLIOGRAFÍA}

Balbín Behrmann, R. de y Bueno Ramírez, P. (1996): "Soto,un ejemplo de Arte Megalítico al Suroeste de la Península". En A. Moure (ed.): "El Hombre fósil" 80 años después. Santander: 466-505.

Bueno Ramírez, P. (1984): "Megalitos en Extremadura". Actas de la mesa Redonda sobre Megalitismo Peninsular. Madrid: 45-50.

- (1987): Megalitismo en Extremadura. Tesis doctoral. Universidad Complutense. Madrid.

- (1987): "Megalitismo en Extremadura: estado de la cuestión”. El Megalitismo en la Península Ibérica. Ministerio de Cultura. Madrid: 72-84.

- (1988): Los dólmenes de Valencia de Alcántara. Excavaciones Arqueológicas en España, 155. Madrid.

- (1989): "Cámaras simples en Extremadura”.XIX Congreso Nacional deArqueología, I: 385-397. Zaragoza.

- (1992): "Les plaques décorées alentejaines: approche de leur étude et analyse". L'Anthropologie, 96, 1: 85110.

- (1994): "La necrópolis de Santiago de Alcántara (Cáceres). Una hipótesis de interpretación para los sepulcros de pequeño tamaño del megalitismo occidental". Boletín del Seminario de Estudios deArte y Arqueología, LX: 25-100.

Bueno Ramírez, P. y Balbín Behrmann, R. de (1992): "L'art mégalithique dans la Péninsule Ibérique. Une vue d'ensemble". L'Anthropologie, 96, 2/3: 499-572.

- (1994): "Estatuas-menhir y estelas antropomorfas en megalitos ibéricos. Una hipótesis de interpretación del espacio funerario". Museo y Centro de Investigaciones de Altamira. Monografías, 17. Santander: $337-$ 347.

- (1995): "La graphie du serpent dans la culture mégalithique péninsulaire. Représentations de plein air et représentations dolméniques". L'Anthropologie,99, 2 / 3: 357-381.

- (1996a): "La decoración del dolmen de Alberite". En F. Ramos y F. Giles (eds.): El dolmen de Alberite (Villamartín). Aportaciones a las formas económicas y sociales de las comunidades neolíticas en el Noroeste de Cádiz. Cádiz-Villamartin: 285-312.

- (1996b): "El papel del elemento antropomorfo en el Arte Megalítico ibérico". Révue archéologique de l'Ouest, Suppl. 8: 41-64.

- (1997a): "Arte Megalítico en el Suroeste de la Península Ibérica, ¿grupos en el Arte Megalítico ibérico?". Saguntum, 30, II: 153-161.

- (1997b): "Ambiente funerario en la sociedad megalítica ibérica: Arte megalítico peninsular". En A. Rodriguez Casal (ed.): O Neolítico atlántico e as orixes do megalitismo. Actas do Coloquio Internacional (Santiago de Compostela, 1996): 695-718.

Calado, M.(1994): “A necrópole dolménica do Monte do Lucas (Terena.Alandroal). Actas das V Jornadas Ar-

T. P., 55, n. $^{\circ} 1,1998$ 
queológicas. Associaçao dos Arqueólogos portugueses, 2: 125-131. Lisboa

Cruz, D. da (1988): "O Megalitismo do Norte de Portugal". Trabalhos de Antropología e Etnología, 28:15-56.

Delibes,G.; Rojo, M. y Represa, I. (1993): Dólmenes de la Lora (Burgos). Junta de Castilla y León. Consejería de Cultura y Turismo. Salamanca.

EnRíQuez, J.J. (1990): El Calcolítico o Edad del Cobre de la cuenca extremeña del Guadiana: los poblados. Museo de Badajoz. Badajoz.

Gomes, M.V. (1997): "Anta da Belhoa (Reguengos de Monsaraz, Evora). Resultados da campanha de escavaçoes de 1992". Cuadernos de Cultura de Reguengos de Monsaraz, 1: 39-69.

JoRGE, V.O. (1990): "Les monuments mégalithiques du Nord du Portugal". Probleme der Megalithgräber- forschung . Madrider Forschungen, Band,16. Berlín: 35-52.

LeISNER, G. (1949): "Antas dos arrededores de Evora". A Cidade de Evora, 15/16-17/18.

Montano, C. (1987): "Aproximación al estudio de los monumentos megalíticos de Alcántara". Anales ADECO. Cáceres: 29-58.

Montano, C. e Iglesias, M. (1988): Grabados rupestres en Alcántara. Cáceres. Ayuntamiento de Alcántara.

Oliveira, J. (1990): "Aspectos do megalitismo do Nordeste alentejano. Análise espacial das sepulturas megalíticas da bacia hidrográfica do río Sever)". Actas do Encontro Regional de Historia. Evora.

- (1994): Sepulturas megalíticas del término municipal de Cedillo-Provincia de Cáceres. Ayuntamiento de Cedillo. 